\title{
An Observational Evidence for the Difference between the Short and Long Gamma-Ray Bursts
}

\author{
L.G. Balázs , P. Mészáros ${ }^{\dagger}$, Z. Bagoly , I. Horváth ${ }^{\ddagger}$ and A. Mészáros ${ }^{\S}$ \\ Konkoly Observatory, Budapest, Box 67, H-1525, Hungary \\ †ept. of Astronomy, Pennsylvania State University, 525 Davey Lab. University Park, PA 16802, USA \\ Lab. for Information Technology, Eötvös University, Budapest, Pázmány Péter sétány 1/A, H-1518, Hungary \\ ${ }^{*}$ Dept. of Physics, Bolyai Military University, BJKMF, Budapest, Box 12, H-1456, Hungary \\ ${ }^{\S}$ Astron. Inst. of the Charles University, 18000 Prague 8, V Holešovičkách 2, Czech Republic
}

\begin{abstract}
.
The intrinsic fluence and duration distributions of gamma-ray bursts are well represented by log-normal distributions. This allows a bivariate log-normal distribution fit to be made to the BATSE short and long bursts separately. A statistically significant difference between the long and short groups is found. We argue that the effect is probably real. Applying the Cramér's theorem these results lead to some predictions for models of long and short bursts.
\end{abstract}

\section{INTRODUCTION}

The simplest grouping of gamma-ray bursts (GRBs) is given by their well-known bimodal duration distribution. This divides bursts into long $\left(T_{90}>2 \mathrm{~s}\right)$ and short $\left(T_{90}<2 \mathrm{~s}\right)$ duration groups [6]. The bursts measured with the BATSE instrument on the Compton Gamma-Ray Observatory are usually characterized by 9 observational quantities, i.e. 2 durations, 4 fluences and 3 peak fluxes [7]. In [1] we used the principal components analysis (PCA) technique to show that these 9 quantities can be reduced to only two significant independent variables, or principal components (PCs). The observational fact, that the dominant principal component consists mainly of the durations and the fluences, may be of consequence for the physical modeling of the burst mechanism.

In this paper we investigate the nature of this principal component decomposition, and in particular, we analyze quantitatively the relationship between the fluences and durations implied by the first PC. We analyze the distribution of the observed fluences and durations of the long and the short bursts, and we present arguments indicating that the intrinsic durations and fluences are well represented by log-normal distributions. The implied bivariate log-normal distribution represents an ellipsoid in these two variables, whose major axis inclinations are statistically different for the long and the short bursts.

Our GRB sample is selected from the Current BATSE Gamma-Ray Burst Catalog according to two criteria, namely, that they have both measured $T_{90}$ durations and fluences (for the definition of these quantities see [7], henceforth referred to as the Catalog). The Catalog in its final version lists 2041 bursts for which a value of $T_{90}$ is given. The fluences are given in four different energy channels, $F_{1} ; F_{2} ; F_{3} ; F_{4}$, whose energy bands correspond to $25 ; 50] \mathrm{keV},[50 ; 100] \mathrm{keV},[100 ; 300] \mathrm{keV}$ and $>300$ $\mathrm{keV}$. The "total" fluence is defined as $F_{t o t}=F_{1}+F_{2}+$ $F_{3}+F_{4}$, and we restrict our sample to include only those GRBs which have $F_{i}>0$ values in at least the channels $F_{1} ; F_{2} ; F_{3}$. Concerning the fourth channel, whose energy band is $>300 \mathrm{keV}$, if we had required $F_{4}>0$ as well, this would have reduced the number of eligible GRBs by ' $20 \%$. Hence, we decided to accept also the bursts with $F_{4}=0$, rather than deleting them from the sample. Using therefore these two cuts, we are left with $N=1929$ GRBs, all of which have defined $T_{90}$ and $F_{t o t}$, as well as peak fluxes $P_{256}$. This is the sample that we study.

\section{FITTING THE LOGARITHMIC FLUENCES AND DURATIONS BY THE SUPERPOSITION OF TWO BIVARIATE DISTRIBUTIONS}

We assume here that the distributions of the variables $T_{90}$ and $F_{t o t}$, for both the short and long groups, can well be approximated by log-normals. As it was already noted in a previous contribution [4], this is an acceptable assumption. In this case it is possible to fit simultane- 
TABLE 1. The best fit parameters of the sum of two bivariate log-normal distributions for $x=\log T_{90}$ and $y=\log F_{\text {tot }}$ for the sample with $N=1929$.

\begin{tabular}{rrrr}
\hline$a_{x 1}$ & -0.08 & $a_{x 2}$ & 1.54 \\
$a_{y 1}$ & -6.22 & $a_{y 2}$ & -5.29 \\
$\sigma_{x 1}^{\prime}$ & 0.73 & $\sigma_{x 2}^{\prime}$ & 0.67 \\
$\sigma_{y 1}^{\prime}$ & 0.46 & $\sigma_{y 2}^{\prime}$ & 0.37 \\
$\tan \alpha_{1}$ & 0.91 & $\tan \alpha_{2}$ & 2.29 \\
$\mathrm{~W}$ & 0.32 & & \\
\hline
\end{tabular}

ously the values of $\log F_{\text {tot }}$ and $\log T_{90}$ by a single twodimensional (bivariate) normal distribution. This distribution has five parameters (two means $a_{x}, a_{y}$, two dispersions $\sigma_{x}, \sigma_{y}$, and the correlation coefficient $r$, where $\left.x=\log T_{90}, y=\log F_{t o t}\right)$. An equivalent set of parameters consists of taking the same two means with two other dispersions $\sigma_{x}^{i} \sigma_{y}^{i}$, and (instead of the correlation coefficient) the angle $\alpha$ between the axis $\log T_{90}$ and the semi-major axis of the "dispersion ellipse". (In the case of bivariate normal distributions, the constant probability curves define ellipses with well-defined axis directions). In this case $\alpha$ and the correlation coefficient are related unambiguously through analytical formulas [5]. If the data are well fitted by this bivariate normal distribution, then the distributions of each of the variables by themselves must also be univariate normal distributions (the marginal distributions are also normal).

A crucial point in this analysis is that, when the $r$ correlation coefficient differs from zero, then the semimajor axis of the dispersion ellipse represents a linear relationship between $\log T_{90}$ and $\log F_{\text {tot }}$, with a slope of $m=\tan \alpha$. This linear relationship between the logarithmic variables implies a power law relation of form $F_{t o t}=$ $\left(T_{90}\right)^{m}$ between the fluence and the duration, where $m$ may be different for the two groups.

Fitting the data with the superposition of two bivariate log-normal distributions can be done by a standard search for 11 parameters with $N=1929$ measured points. (Both log-normal distributions have five parameters; the eleventh parameter defines the weight of the first log-normal distribution.) We will use $\tan \alpha$ as the fifth parameter for both partial distributions ("terms"). Figure 1 . shows the values of $x=\log T_{90}$ and $y=\log F_{t o t}$ for the $N=1929$ GRBs. Each GRB defines a point in the $x ; y$ plane with coordinates $x_{i} ; y_{i}(i=1 ; 2 ;:: ; N)$. The theoretical curve is a sum of two normal distributions. The normalization constant of the first [second] term is $N W[N(1 \quad W)]$, where $W$ is the weight $(0$ $W$ 1). For the first (second) term the parameters are $a_{x 1} ; a_{y 1} ; \sigma_{x 1} ; \sigma_{y 1} ; \alpha_{1}\left(a_{x 2} ; a_{y 2} ; \sigma_{x 2} ; \sigma_{y 2} ; \alpha_{2}\right)$.

We obtain the best fit to the 11 parameters through a maximum likelihood (ML) estimation. The results are collected at Table 1.

\section{DISCUSSION AND CONCLUSION}

We have presented evidence indicating that there is a power law relationship between the logarithmic fluences and $\log T_{90}$ of the GRBs in the Current BATSE Catalog, based on a maximum likelihood estimation of the parameters of the bivariate distribution of these measured quantities.

An intriguing corollary of these results is that the exponents in the power law dependence between fluence and duration differ significantly for the two groups of short $\left(T_{90}<2 \mathrm{~s}\right)$ and long $\left(T_{90}>2 \mathrm{~s}\right)$ bursts.

These two results may have an interesting impact on the models of GRBs.

As it was already discussed in [4], the application of the mathematical Cramér's theorem [3,8] ensures that there is also the same power law relationship between the total emitted energies and the intrinsic durations. Because the exponents are different for the short and long subgroups, these subgroups should also be generated by different scenarios.

This results, together with the conclusion that for the short (long) bursts the total released energy is proportional to the intrinsic duration (to the square of intrinsic duration) were already announced by [4].

Nevertheless, a care is needed yet. According to the Bayes theorem [8], the probability density $P\left(F_{t o t} ; T_{90}\right)$ of fluence and $T_{90}$ is given by

$$
P\left(F_{t o t} ; T_{90}\right)=\int_{0}^{\infty} P\left(F_{t o t} ; T_{90} P\right) G(P) d P ;
$$

where $P$ denotes the peak-flux (either on $64 \mathrm{~ms}$, or $256 \mathrm{~ms}$ or $1024 \mathrm{~ms}$ trigger), $G(P)$ is the probability density of $P$, and $P\left(F_{t o t} ; T_{90} P\right)$ is the so-called kernel. (The meaning of $P\left(F_{t o t} ; T_{90}\right)$ is straightforward: $N P\left(F_{\text {tot }} ; T_{90}\right) d F_{\text {tot }} d T_{90}$ defines the number of GRBs in the intervals $\left[F_{\text {tot }} ;\left(F_{\text {tot }}+d F_{\text {tot }}\right)\right]$ and $\left[T_{90} ;\left(T_{90}+d T_{90}\right)\right.$, respectively, where $N$ is the number of GRBs.) Unfortunately, $G(P)$ is well biased by instrumental effects, and - to have the real intrinsic biasfree relation between the fluence and duration - the kernel should be known. Of course, in principle, due to the biases in $G(P)$, it is not sure that the the observed relation between the fluence and $T_{90}$ and the relation coming from the kernel are identical.

We proceeded a new estimation of these phenomena by different approximations of kernel, and - it seems our previous proclamations further hold. The details of our studies will be published elsewhere [2].

\section{ACKNOWLEDGMENTS}

This research was supported in part through OTKA grants T024027 (L.G.B.), F029461 (I.H.) and T034549; 


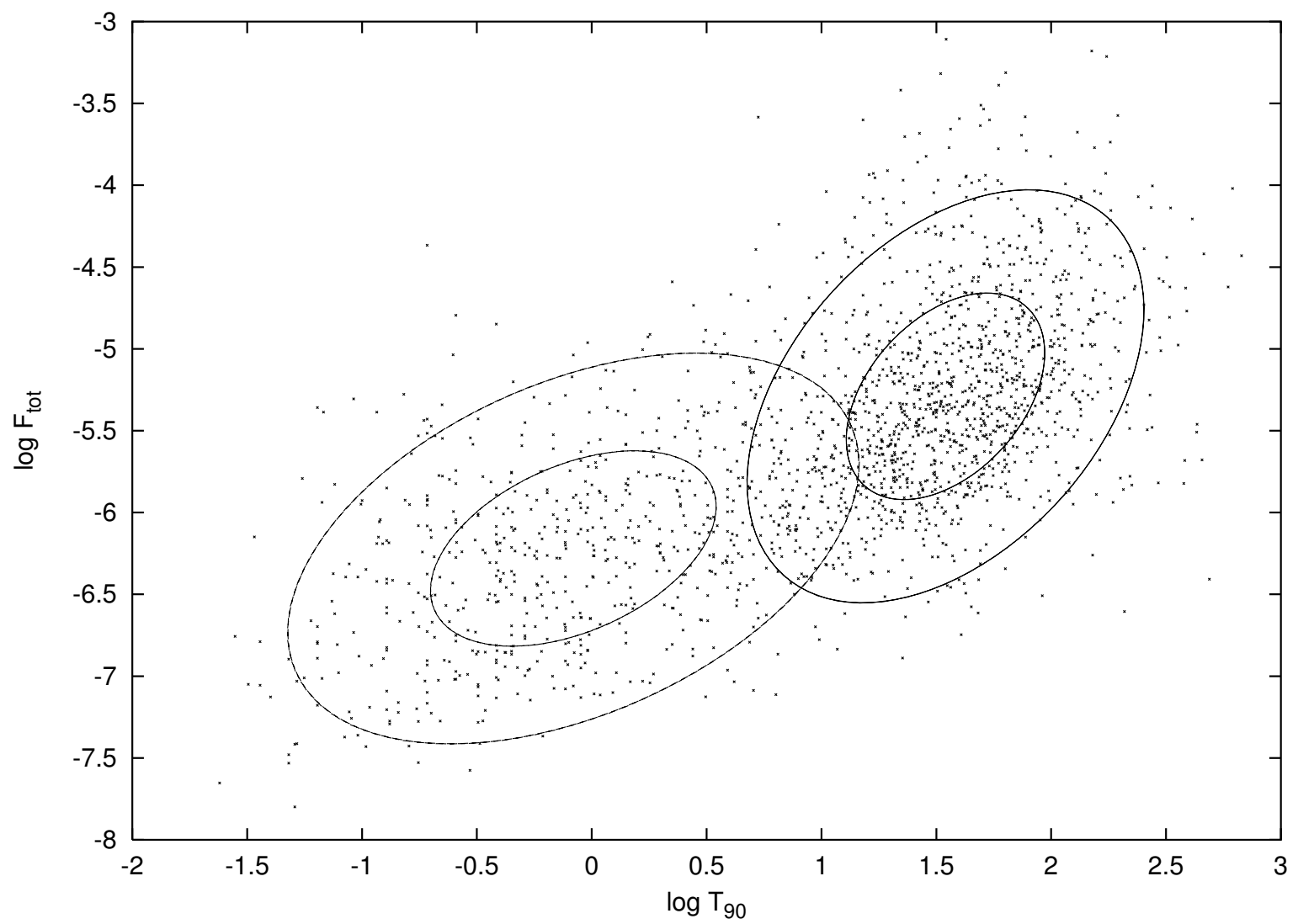

FIGURE 1. The best fit of two bivariate log-normal distributions for the whole BATSE sample (1929 GRBs). The ellipses give the $1 \sigma$ and $2 \sigma$ probabilities.

NASA grant NAG5-2857, Guggenheim Foundation and Sackler Foundation (P.M.); and Czech Research Grant J13/98: 113200004 (A.M.).

\section{REFERENCES}

1. Bagoly, Z., Mészáros, A., Horváth, I., Balázs, L.G., and Mészáros, P., ApJ, 498, 342, 1996.

2. Balázs, L.G., Mészáros, P., Bagoly, Z., Horváth, I., Mészáros, A., in preparation, 2002.

3. Cramér, H., Random variables and probability distributions, Cambridge Tracts in Mathematics and Mathematical Physics, No.36, Cambridge University Press, Cambridge, 1937.

4. Horváth, I., Balázs, L.G., Mészáros, P., Bagoly, Z., Mészáros, A., in Proceedings of Second Roma Workshop on GRBs, ed. N. Masetti, Springer, in press.

5. Kendall, M. and Stuart, A., The Advanced Theory of Statistics, Griffin, London, 1976.

6. Kouveliotou, C., et al., ApJ, 413, L101. 1993.

7. Meegan, C.A., et al., Current BATSE Gamma-Ray Burst Catalog, (2000) URL http: / / gammaray.msfc. nasa.gov/batse/grb/catalog/current.
8. Rényi, A. Wahrscheinlichtkeitsrechnung, VEB Deutscher Verlag der Wissenschaften, Berlin, 1962 